Pacific Journal of Mathematics

CONVERGENCE IN SPACES OF SUBSETS 


\title{
CONVERGENCE IN SPACES OF SUBSETS
}

\author{
R. J. GAZIK
}

\begin{abstract}
Under certain conditions on a class $\mathscr{C}$ of subsets of either a uniform convergence space, uniform space, or bounded metric space, a natural convergence structure for $\mathscr{C}$ is defined which is, respectively, $u$-uniformizable, uniformizable, metrizable. Conditions which are sufficient for the convergence structure to be separated, topological, regular, are given. In the uniform space case some convergence properties of $\mathscr{C}$ are investigated and a fixed point theorem is proved for certain $\mathscr{C}$-multifunctions.
\end{abstract}

1. Introduction. In order to establish notation and provide some motivation we will, in this section, review a few basic results which deal with uniform convergence structures. The reader is assumed to be familiar with the very basic theorems from the theory of convergence spaces [5].

In order to obtain concepts like Cauchy filter, uniform convergence, total boundedness, which were previously defined only in uniform spaces, Fischer and Cook began the study of uniform convergence spaces in [4]. A uniform convergence structure $\Sigma$ on a set $E$ is an intersection ideal in the collection of filters on $E \times E$ which satisfies the following axioms:

$\left(U_{1}\right)$ The filter of supersets of the diagonal in $E \times E$ is a member of $\Sigma$.

$\left(U_{2}\right)$ If $\mathscr{G} \in \Sigma$, so its inverse.

$\left(U_{3}\right)$ If $\mathscr{C}, \mathscr{F} \in \Sigma$ and the composition filter $\mathscr{G} \circ \mathscr{F}$ exists, then it belongs to $\Sigma$.

A uniform convergence space $(E, \Sigma)$ is a set $E$ along with a convergence structure $\Sigma$ on $E$. A convergence structure $\sigma(\Sigma)$ is induced on $E$ in a natural way: define $\mathscr{F} \in \sigma(\Sigma)(x)$ if and only if $\mathscr{F} \times \dot{x} \in$ $\Sigma$. If $P$ is a property which can be defined by convergence (for instance compactness, regularity, Hausdorffness, etc.) then, by definition, $(E, \Sigma)$ has property $P$ if and only if $\sigma(\Sigma)$ has it. Also, most definitions of uniform properties are available in uniform convergence spaces and are generalizations of the uniform topology case. For example, a filter $\mathscr{F}$ on $E$ is a Cauchy filter if $\mathscr{F} \times \mathscr{F} \in \Sigma ;(E, \Sigma)$ is complete if each Cauchy filter converges with respect to $\sigma(\Sigma) ;(E$, $\Sigma)$ is totally bounded if each filter on $E$ is coarser than a Cauchy filter; a map $f$ between uniform convergence spaces $(E, \Sigma),(F, \psi)$ is uniformly continuous on $E$ if $(f \times f) \Sigma \subset \psi$.

With these definitions one obtains results which, for the most 
part, parallel the uniform space case. For example, it is true that each uniform convergence space has a completion [8], and that a uniform convergence space is compact if and only if it is complete and totally bounded [4]. The following result is due, independently, to Keller [6] and Cochran [3].

THEOREM 1.1. Each Hausdorff convergence space $(E, \delta)$ is $u$ uniformizable. That is, there exists a uniform convergence structure $\Sigma$ on $E$ such that $\delta=\sigma(\Sigma)$.

If $(E, \Sigma)$ is a uniform convergence space, a subset $\psi$ of $\Sigma$ is a base for $\Sigma$ if each member of $\Sigma$ is finer than a member of $\psi$. The following result (see [4]) shows the relationship between uniform convergence spaces and uniform spaces.

THEOREM 1.2. If a uniform convergence structure $\Sigma$ for $E$ has a base consisting of exactly one filter $\mathscr{U}$ then $\mathscr{U}$ is a uniform structure for $E$; each uniform structure $\mathscr{Q}$ for $E$ is a base of exactly one

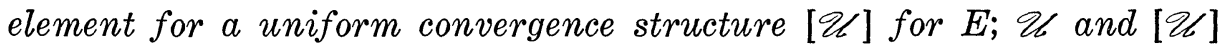
have exactly the same set of Cauchy filters and exactly the same set of convergent filters.

Now consider the following well known construction: If $(E, \mathscr{C})$ is a uniform space and $\mathscr{C}$ is the class of nonempty, closed subsets of $E$, then a uniform structure for $\mathscr{C}$ is generated by sets of the form $\{(A, B): A, B \in \mathscr{C}, A \subset U(B), B \subset U(A)\}, U \in \mathscr{Q}$. It follows that a filter $\mathscr{F}$ on $\mathscr{C}$ converges to $A \in \mathscr{C}$, with respect to the completely regular topology on $\mathscr{C}$ induced by $\mathscr{W}$, if and only if for each $U \in \mathscr{C}$, there exists $\mathscr{F} \in \mathscr{F}$ such that $F \subset V(A)$ and $A \subset V(F)$ for each $F \in \mathscr{F}$. The topology induced on $\mathscr{C}$ is called the uniform topology on $\mathscr{C}$ ]7].

The remarks above motivate the consideration of convergence of sets of a class $\mathscr{C}$ (of not necessarily closed sets) in any space where "closeness of sets" is meaningful. We will begin the discussion with uniform convergence spaces. According to Theorem 1.1, these include Hausdorff topological spaces and many others which are not topological spaces.

2. Convergence classes. For the remainder of this section a uniform convergence space $(E, \Sigma)$ will be a set $E$ along with a base $\Sigma$ for a uniform convergence structure on $E$.

Definition 2.1. Let $(E, \Sigma)$ be a uniform convergence space. A nonempty class $\mathscr{C}$ of nonempty subsets of $E$ is called a convergence 
class for $(E, \Sigma)$ if and only if $A \subset V(A)$ for each $A \in \mathscr{C}, V \in \mathscr{J}$, $\mathscr{J} \in \Sigma$.

DEFINITION 2.2. Let $(E, \Sigma)$ be a uniform convergence space and let $\mathscr{C}$ be a nonempty collection of nonempty subsets of $E$. The function $\tau(\mathscr{C})$ from $\mathscr{C}$ into the power set of the filters on $\mathscr{C}$ is defined by $\mathscr{F} \in \tau(\mathscr{C})(A)$ if and only if for each $V \in \mathscr{J}, \mathscr{J} \in \Sigma$, there exists $\mathscr{F} \in \mathscr{F}$ such that $F \subset V(A)$ and $A \subset V(F)$ for each $F \in \mathscr{F}$.

THEOREM 2.1. The function $\tau(\mathscr{C})$ of Definition 2.2 is a convergence structure on $\mathscr{C}$ if and only if $\mathscr{C}$ is a convergence class for $(E, \Sigma)$.

Proof. It is clear that if $\mathscr{F} \in \tau(\mathscr{C})(A)$ and $\mathscr{G}$ is finer than $\mathscr{F}$, then $\mathscr{G} \in \tau(\mathscr{C})(A)$. If $\mathscr{F}, \mathscr{G} \in \tau(\mathscr{C})(A)$ then, since $\{\mathscr{F} \cup \mathscr{G}: \mathscr{F} \in$ $\mathscr{T}, \mathscr{G} \in \mathscr{G}\}$ is a base for $\mathscr{G} \wedge \mathscr{G}, \mathscr{F} \wedge \mathscr{G} \in \tau(\mathscr{C})(A)$. Hence, $\tau(\mathscr{C})$ is a convergence structure for $\mathscr{C}$ if and only if the ultrafilter generated by $A$ is in $\tau(\mathscr{C})(A)$ for each $A \in \mathscr{C}$. But this is equivalent to the statement that $A \subset V(A)$ for each $A \in \mathscr{C}, V \in \mathscr{J}, \mathscr{J} \in \Sigma$.

Some additional properties which may sometimes be required of a convergence class $\mathscr{C}$ for a uniform convergence space $(E, \Sigma)$ are: $A \subset B$.

$\left(A_{1}\right)$ If $A, B \in \mathscr{C}$ and $A \subset V(B)$ for each $V \in \mathscr{J}, \mathscr{J} \in \Sigma$, then

$\left(A_{2}\right)$ If $A, B \in \mathscr{C}$ and $A \subset V(B), B \subset V(A)$ for each $V \in \mathscr{J}, \mathscr{J} \in$ $\Sigma$, then $A=B$.

$\left(A_{3}\right)$ For each $\mathscr{J} \in \Sigma$ and $V \in \mathscr{J}$, there exists $U \in \mathscr{J}$ such that $U^{2}(A) \subset V(A)$ for all $A \in \mathscr{C}$.

$\left(A_{4}\right)$ For each $\mathscr{J} \in \Sigma, V \in \mathscr{J}, A \in \mathscr{C}$, there exists $U \in \mathscr{J}$ such that $U^{2}(A) \subset V(A)$.

$\left(A_{5}\right)$ If $A, B \in \mathscr{C}$, then $A \cup B \in \mathscr{C}$.

THEOREM 2.2. Let $\mathscr{C}$ be a convergence class for the uniform convergence space $(E, \Sigma)$. Then

(1) If either $\left(A_{1}\right)$ and $\left(A_{4}\right)$ or $\left(A_{2}\right)$ and $\left(A_{4}\right)$ hold, then $\tau(\mathscr{C})$ is separated and u-uniformizable.

(2) If $\left(A_{5}\right)$ holds and $\tau(\mathscr{C})$ is separated, then $\left(A_{1}\right)$ and $\left(A_{2}\right)$ hold.

(3) $\left(A_{1}\right)$ implies $\left(A_{2}\right)$ and, if $\left(A_{5}\right)$ holds, $\left(A_{2}\right)$ implies $\left(A_{1}\right)$.

Proof. (1) Suppose $\mathscr{T} \in \tau(\mathscr{C})(A) \cap \tau(\mathscr{C})(B)$ and let $V \in \mathscr{J}, \mathscr{J} \in \Sigma$. By $\left(A_{4}\right)$ there exist $U, W \in \mathscr{J}$ such that $U^{2}(B) \subset V(B), W^{2}(A) \subset V(A)$. Then $S=U \cap W \in \mathscr{J}$ so $F \subset S(A), A \subset S(F), B \subset S(F), F \subset S(B)$ for all $F \in \mathscr{F}$ and some $\mathscr{F} \in \mathscr{F}$. From these relations, $A \subset U^{2}(B) \subset V(B)$ and $B \subset W^{2}(A) \subset V(A)$ so, if either $\left(A_{1}\right)$ or $\left(A_{2}\right)$ hold, $A=B$; that is, $\tau(\mathscr{C})$ is separated. It follows from Theorem 1.1 that $\tau(\mathscr{C})$ is $u$-uniformizable. 
(2) Suppose $A \subset V(B)$ for all $V \in \mathscr{J}, \mathcal{J} \in \Sigma$. Then $A \cup B \subset V(B)$ and $B \subset V(A \cup B)$ so, since $\left(A_{5}\right)$ holds, the ultrafilter generated by $B$ converges to $A \cup B$. Since $\tau(\mathscr{C})$ is separated $B=A \cup B$ so $A \subset B$. A similar argument shows that $\left(A_{2}\right)$ holds.

(3) If $\left(A_{1}\right)$ holds, $\left(A_{2}\right)$ holds. If $A \subset V(B)$ for all $V \in \mathscr{J}, \mathscr{J} \in$ $\Sigma$, then, since $A \cup B \in \mathscr{C}, A \cup B \subset V(B)$ and $B \subset V(A \cup B)$; it follows that $A \cup B=B$ and $A \subset B$.

THEOREM 2.3. Let $\mathscr{C}$ be a convergence class for a uniform convergence space $(E, \Sigma)$. If $\left(A_{3}\right)$ holds then $\tau(\mathscr{C})$ is a topological space; that is, there is a topology $\sigma(\mathscr{C})$ on $\mathscr{C}$ such that a filter $\mathscr{F}$ converges to $A \in \mathscr{C}$ with respect to $\sigma(\mathscr{C})$ if and only if $\mathscr{F} \in \tau(\mathscr{C})(A)$.

Proof. It suffices to show that if $\mathscr{F} \notin \tau(\mathscr{C})(A)$, then there exists $\mathscr{X}$, a subset of $\mathscr{C}$, such that $A \in \mathscr{X}, \mathscr{X} \notin \mathscr{F}$ and if $B \in \mathscr{X}, \mathscr{G} \in$ $\tau(\mathscr{C})(B)$, then $\mathscr{X} \in \mathscr{G}$.

Now suppose $\mathscr{T} \notin \tau(\mathscr{C})(A)$. Then for some $V \in \mathscr{J}, \mathscr{J} \in \Sigma$, no $\mathscr{F} \in \mathscr{F}$ satisfies

(1) $F \in \mathscr{F}$ implies $F \subset V(A)$ and $A \subset V(F)$.

Define a subset $\mathscr{X}$ of $\mathscr{C}$ as follows: $\mathscr{X}$ consists of all $B \in \mathscr{C}$ such that

(2) $B \subset V(B)$, and $A \subset V(B)$, and

(3) there exists $U \in \mathscr{J}$ such that if $H \in \mathscr{C}$ and $H \subset U(B)$ and $B \subset U(H)$, then $H \subset V(A)$ and $A \subset V(H)$.

Now $A \in \mathscr{Q}$ for $A \subset V(A)$ and we may take the $U$ required by (3) to be $V$. $\mathscr{X} \notin \mathscr{F}$ by (1) and (2).

Suppose now that $\mathscr{G} \in \tau(\mathscr{C})(B), B \in \mathscr{X}$. We show $\mathscr{E} \in \mathscr{G}$ by proving that $\mathscr{X}$ contains a member of $\mathscr{G}$.

Since $B \in \mathscr{X}$, condition (2) holds for some $U \in \mathscr{J}$. By $\left(A_{3}\right)$ there exists $W \in \mathscr{J}$ such that $W^{2}(D) \subset U(D)$ for all $D \in \mathscr{C}$. Since $\mathscr{G} \in$ $\tau(\mathscr{C})(B)$, there exists $\mathscr{G} \in \mathscr{G}$ such that $G \subset W(B)$ and $B \subset W(G)$ for all $G \in \mathscr{C}$.

Let $G \in \mathscr{G}$. Since $B \in \mathscr{Z}$ and $G \subset W(B), B \subset W(G)$, then $G \subset V(A)$ and $A \subset V(G)$ so $G$ satisfies (2).

Suppose $H \in \mathscr{C}$ and $H \subset W(G), G \subset W(H)$. Then $H \subset W^{2}(B) \subset U(B)$ and $B \subset W(G) \subset W^{2}(H) \subset U(H)$ so, since $B \in \mathscr{P}, H \subset V(A)$ and $A \subset V(H)$. This shows that each $G \in \mathscr{G}$ satisfies (3).

In summary, $\mathscr{G} \subset \mathscr{X}, \mathscr{G} \in \mathscr{G}$, so $\mathscr{X} \in \mathscr{G}$.

THeOREM 2.4. With the same assumptions as in Theorem 2.3, the topological space $(\mathscr{C}, \sigma(\mathscr{C}))$ is regular.

Proof. Recall first that a net in a topological space converges to a point if and only if its filter of final sections converges to the 
same point. In the present context it follows from the previous theorem that a net $\left(A_{n}: n \in D\right)$ in $\mathscr{C} \sigma(\mathscr{C})$-converges to $A \in \mathscr{C}$ (written $\left.\left(A_{n}: n \in D\right) \rightarrow A\right)$ if and only if for each $V \in \mathscr{J}, \mathscr{J} \in \Sigma, A_{n} \subset V(A)$ and $A \subset V\left(A_{n}\right)$ for $n$ sufficiently large.

Now let $\left(A_{i j}: i \in I, j \in J_{i}\right)$ be a simply convergent double net in $\mathscr{C}$ with $\left(A_{i j}: j \in J_{i}\right) \rightarrow P_{i} \in \mathscr{C}$ for each $i \in I$. Let $h$ be the diagonal net on $T=I \times \Pi\left(J_{i}: i \in I\right)$ defined by $h(i, g)=A_{i, g(i)}$ and suppose the diagonal net converges to $X \in \mathscr{C}$. We prove $(\mathscr{C}, \sigma(\mathscr{C}))$ is regular by showing that $\left(P_{i}: i \in I\right) \rightarrow X$.

Let $V \in \mathscr{J}, \mathscr{J} \in \Sigma$. By $\left(A_{3}\right)$ there exists $U \in \mathscr{J}$ such that $U^{2}(B) \subset$ $V(B)$ for all $B \in \mathscr{C}$. Since the diagonal net converges to $X$,

(1) $A_{i, g(i)} \subset U(X), X \subset U\left(A_{i, g(i)}\right)$ for $(i, g) \geqq\left(i_{0}, g_{0}\right)$. Since each $\left(A_{i j}: j \in J_{i}\right) \rightarrow P_{i}$,

(2) $A_{i j} \subset U\left(P_{i}\right), P_{i} \subset U\left(A_{i j}\right)$ for each $i \geqq i_{0}$ and $j \geqq j(i, V)$. Define $w \in \Pi\left(J_{i}: i \in I\right)$ by requiring $w(i)$ to be greater than or equal to both $g_{0}(i), j(i, V)$ if $i \geqq i_{0}$ and $w(i)=g_{0}(i)$ otherwise. Then, for $i \geqq i,(i, w) \geqq$ $\left(i_{0}, g_{0}\right)$ so by (1), $A_{i, w(i)} \subset U(X)$ and $X \subset U\left(A_{i, w(i)}\right)$. By (2) $A_{i w(i)} \subset U\left(P_{i}\right)$ and $P_{i} \subset U\left(A_{i, w(i)}\right)$. Hence, for $i \geqq i_{0}, P_{i} \subset U^{2}(X) \subset V(X)$ and $X \subset U^{2}\left(P_{i}\right) \subset$ $V\left(P_{i}\right)$. It follows that $\left(P_{i}: i \in I\right) \rightarrow X$ and $(\mathscr{C}, \sigma(\mathscr{C}))$ is regular.

It should be pointed out that a number of other natural convergences on a convergence class $\mathscr{C}$ might be studied. The following are a few such examples.

(1) $\mathscr{F} \in \psi(\mathscr{C})(A)$ if and only if there exists $\mathscr{J} \in \Sigma$ such that: for each $V \in \mathscr{J}$ there is an $\mathscr{F} \in \mathscr{F}$ such that $F \subset V(A)$ and $A \subset V(F)$ for each $F \in \mathscr{F}$.

(2) $\mathscr{F} \in \lambda(\mathscr{C})(A)$ if and only if for each $V \in \mathscr{J}, \mathscr{J} \in \Sigma, a \in A$, there exists $\mathscr{F} \in \mathscr{F}$ such that $F \subset V(A)$ and $F \cap V(\alpha) \neq \phi$ for each $F \in \mathscr{F}$.

(3) $\mathscr{F} \in \alpha(\mathscr{C})(A)$ if and only if there exists $\mathscr{J} \in \Sigma$ such that: for each $V \in \mathscr{J}, a \in A$, there exists $\mathscr{F} \in \mathscr{F}$ such that $F \subset V(A)$ and $F \cap V(a) \neq \phi$ for each $F \in \mathscr{F}$.

EXAMPLE 1. Let $\Sigma$ consist of just one uniform structure $\mathscr{f}$ for $E$ and let $\mathscr{C}$ be any nonempty class of nonempty subsets of $E$. By Theorem $1.2 \Sigma$ is a base for a uniform convergence structure on E. Clearly $A \subset V(A)$ for each $V \in \mathscr{J}, A \in \mathscr{C}$, so $\mathscr{C}$ is a convergence class for $(E, \Sigma)$. In particular, if $\mathscr{C}$ is the class of nonempty closed subsets of $E$, then, by the discussion at the end of $\S 1, \tau(\mathscr{C})$ convergence is precisely the convergence of closed sets in the uniform topology on $\mathscr{C}$. (See [7].)

ExAmple 2. Let $E$ be a Hausdorff topological space and, for each finite subset $S$ of $E$, define $\mathscr{J}(S)$ to be the filter $\wedge(\mathscr{N}(x) \times \mathscr{N}(x)$ : $x \in S) \wedge \mathscr{D}$, where $\mathscr{D}$ is the filter of supersets of the diagonal in 
$E \times E$ and $\mathscr{N}^{\prime}(x)$ is the neighborhood filter at $x$. The collection $\Sigma=\{\mathscr{J}(S): S$ is a finite subset of $E\}$ is a base for a uniform convergence structure on $E$. It is not hard to see that the convergence induced by $\Sigma$ is precisely convergence in the topological space $E$. Each member of each $\mathscr{J}(S)$ contains the diagonal so any $\mathscr{C}$ is a convergence class for $(E, \Sigma)$.

If $E$ is a closed interval of real numbers, $\mathscr{C}$ the class of nonempty closed subsets of $E$ and $\mathscr{J}$ the usual uniform structure on $E$, then, by Example 1 and results from [7], $(\mathscr{C}, \tau(\mathscr{C}))$ is compact with respect to the base $\{\mathscr{J}\}$. Now the base $\Sigma$ of Example 2 induces the same convergence on $E$ as does $\{\mathscr{J}\}$, but $(\mathscr{C}, \tau(\mathscr{C}))$ is not compact with respect to $\Sigma$.

Question. If $\mathscr{C}$ is the class of nonempty, closed subsets of a compact uniform convergence space $(E, \Sigma)$, is there a base $\Phi$ for a uniform convergence structure on $E$ such that $\Sigma$ and $\Phi$ induce the same convergence on $E$, and $(\mathscr{E}, \tau(\mathscr{C}))$ is compact with respect to $\Phi$ ?

ExAmple 3. Let $E$ be a Hausdorff topological space and $\mathscr{C}$ any collection of nonempty subsets of $E$. Define $\mathscr{J}$ to be the filter generated by sets of the form $\cup\left(G_{i} \times G_{i}: i \in I\right)$ where $I$ is finite, each $G_{i}$ is open and $\cup\left(G_{i}: i \in I\right)=E$. The collection $\Sigma=\left\{\mathscr{J}, \mathcal{J}^{2}, \mathcal{J}^{3}, \cdots\right\}$ is a base for a convergence structure on $E$ and $\mathscr{C}$ is a convergence class for $(E, \Sigma)$. The topological convergence on $E$ is generally not the same as that induced by $\Sigma$. In this case $\mathscr{F} \in \tau(\mathscr{C})(A)$ if and only if for each $V \in \mathscr{J}$ and each natural number $n$, there exists $\mathscr{F} \in \mathscr{F}$ such that $F \subset V^{n}(A)$ and $A \subset V^{n}(F)$ for each $F \in \mathscr{F}$.

ExAmple 4. Let $E$ be a regular, Hausdorff topological space, $\mathscr{C}$ the class of nonempty, closed subsets of $E$ and $\Sigma$ the base of the previous example. Then $\lambda(\mathscr{C})$ convergence is precisely the convergence of closed sets defined by Choquet on p. 90 of [2].

Question. If $E$ is a topological space, $\mathscr{C}$ its convergence class of closed sets, is there a base $\Sigma$ for a uniform convergence structure on $E$ such that one of the natural convergences $\tau(C), \lambda(\mathscr{C})$, etc. induces the convergence defined by Choquet on p. 87 of [2]?

Of course, the meaning of $\tau(\mathscr{C}), \psi(\mathscr{C}), \lambda(\mathscr{C})$ or $\alpha(\mathscr{C})$ convergence is known as soon as a base for a uniform convergence structure is given. In this regard, see [3] for an explicit construction of a uniform convergence structure for an arbitrary Hausdorff convergence space, and see [4] for construction of natural uniform convergence structures on function spaces. 
3. Convergence classes for uniform spaces. Let $(E, \mathscr{J})$ be a uniform space and let $\mathscr{C}$ be any nonempty class of nonempty subsets of $E$. Since $A \subset V(A)$ for $V \in \mathscr{J}$ and $\left(A_{3}\right)$ of $\S 2$ holds, $\mathscr{C}$ is a convergence class for $(E, \mathscr{J})$ and $\tau(\mathscr{C})$ induces a regular topology $\sigma(\mathscr{C})$ on $\mathscr{C}$. A net $\left(A_{n}: n \in D\right)$ in $\mathscr{C} \sigma(\mathscr{C})$-converges to $A \in \mathscr{C}$ if and only if for each $V \in \mathcal{J}, A_{n} \subset V(A)$ and $A \subset V\left(A_{n}\right)$ for $n$ sufficiently large. In fact, we have the following:

Theorem 3.1. If $(E, \mathcal{J})$ is a uniform space and $\mathscr{C}$ is a nonempty collection of nonempty subsets of $E$, then the topological space $(\mathscr{C}, \sigma(\mathscr{C}))$ is uniformizable.

Proof. For each $V \in \mathscr{J}$, define $\mathscr{T}(V)=\{(A, B): A, B \in \mathscr{C}, A \subset$ $V(B), B \subset V(A)\}$. Then each $\mathscr{T}(V)$ contains the diagonal in $\mathscr{C} \times \mathscr{C}$ and the inverse of $\mathscr{T}(V)$ is itself. Also $\mathscr{T}(V) \supset \mathscr{T}(U) \circ \mathscr{T}(U)$ if $U \circ U \subset V$. Thus $\mu(\mathscr{C})$, the filter generated by the $\mathscr{T}(V)$ 's, is a uniform structure for $\mathscr{C}$. But, from the definitions and the remarks preceding the theorem, a net $\sigma(\mathscr{C})$-converges to $A \in \mathscr{C}$ if and only if it converges to $A$ with respect to the topology generated by $\mu(\mathscr{C})$.

Some additional axioms which may sometimes be required of a convergence class $\mathscr{C}$ for a uniform space $(E, \mathscr{J})$ are:

$\left(B_{1}\right)$ If $A, B \in \mathscr{C}$, then $A \cup B \in \mathscr{C}$.

$\left(B_{2}\right)$ If $A \subset \operatorname{clos} B$, then $A \subset B$.

$\left(B_{3}\right)$ If $A \subset \operatorname{clos} B$ and $B \subset \operatorname{clos} A$, then $A=B$.

$\left(B_{4}\right)$ If $S$ is linearly ordered and $\left(A_{n}: n \in S\right)$ is a decreasing net in $\mathscr{C}\left(n \geqq m\right.$ implies $\left.A_{n} \subset A_{m}\right)$ such that $\cap A_{n} \neq \phi$, then any net $\left(x_{n}\right.$ : $n \in R$ ) with $R$ cofinal in $S$ and $x_{n} \in A_{n}$ for $n \in R$, which converges, converges to a point in $\operatorname{clos}\left(\cap A_{n}\right)$.

THEOREM 3.2. If $\mathscr{C}$ is a convergence class for a uniform space $(E, \mathscr{J})$, then

(1) If $\left(B_{2}\right)$ or $\left(B_{3}\right)$ is satisfied, $(\mathscr{C}, \mu(\mathscr{C}))$ is Hausdorff.

(2) If $(\mathscr{C}, \mu(\mathscr{C}))$ is Hausdorff and $\left(B_{1}\right)$ holds, then $\left(B_{2}\right)$ and $\left(B_{3}\right)$ hold.

(3) $\left(B_{2}\right)$ implies $\left(B_{3}\right)$ and, if $\left(B_{1}\right)$ holds, $\left(B_{3}\right)$ implies $\left(B_{2}\right)$.

Proof. This follows from Theorem 2.2.

ExAmple 5. A simple example of a convergence class $\mathscr{C}$ for a uniform space $(E, \mathscr{J})$ for which $(\mathscr{C}, \mu(\mathscr{C}))$ is Hausdorff and $\mathscr{C}$ does not consist of closed sets is obtained by taking $\mathscr{C}$ to be the class of all nonempty, regular open subsets of $E$. Recall that an open set $G$ is regular open if $G=\operatorname{Int}(\operatorname{clos} G)$. It is clear, then, that $\mathscr{C}$ satisfies $\left(B_{3}\right)$ so $(\mathscr{C}, \mu(\mathscr{C}))$ is Hausdorff. 
DEFINITION 3.1. A net $\left(A_{n}: n \in D\right)$ in $\mathscr{C}$ is increasing (decreasing) if $D$ is totally ordered and $n \geqq m$ implies $A_{n} \supset A_{m}\left(A_{m} \supset A_{n}\right)$.

TheOREm 3.3 Let $(E, \mathscr{J})$ be a compact uniform space and $\mathscr{E}^{3}$ a convergence class for $(E, \mathcal{J})$. Then

(1) An increasing net $\left(A_{n}: n \in D\right)$ in $\mathscr{C}$ converges if and only if there exists $A \in \mathscr{C}$ such that $\cup A_{n} \subset \operatorname{clos} A$ and $A \subset \operatorname{clos}\left(\cup A_{n}\right)$.

(2) If $\left(A_{n}: n \in D\right)$ is a decreasing net in $\mathscr{C}, \cap A_{n} \neq \phi$ and $\left(B_{4}\right)$ is satisfied, then $\left(A_{n}: n \in D\right)$ converges if and only if there exists $A \in$ $\mathscr{C}$ such that $A \subset \operatorname{clos}\left(\cap A_{n}\right)$ and $\cap A_{n} \subset \operatorname{clos} A$.

Proof. A proof of (1) is given. The proof of (2) is similar. If $\left(A_{n}\right.$ : $n \in D) \rightarrow A$ then, if $V \in \mathscr{J}, A_{n} \subset V(A)$ and $A \subset V\left(A_{n}\right)$ for $n$ sufficiently large. But since $\left(A_{n}: n \in D\right)$ is increasing, $\cup A_{n} \subset V(A)$ and $A \subset V\left(\cup A_{n}\right)$. Since $V$ was arbitrary, $\cup A_{n} \subset \operatorname{clos} A$ and $A \subset \operatorname{clos}\left(\cup A_{n}\right)$.

Now suppose $A \in \mathscr{C}$ exists which satisfies $\cup A_{n} \subset \operatorname{clos} A$ and $A \subset$ $\operatorname{clos}\left(\cup A_{n}\right)$. Then, for $V \in \mathscr{J}, n \in D, A_{n} \subset V(A)$. Thus, to show $\left(A_{n}\right.$ : $n \in D) \rightarrow A$ it suffices to show that $A \subset V\left(A_{n}\right)$ for some $n \in D$.

Suppose this is not so. Then there are points $y_{n} \in A-V\left(A_{n}\right)$. The net $\left(y_{n}: n \in D\right)$ has a convergent subnet by the compactness of $(E, \mathscr{J})$. Clearly, the subnet converges to a point $x \in \operatorname{clos} A \subset \operatorname{clos}\left(\cup A_{n}\right)$. If $U^{2} \subset V$, then $U(x) \cap A_{n} \neq \phi$ for $n$ sufficiently large. But $\left(y_{n}: n \in D\right)$ is frequently in $U(x)$ so there is an index $n \in D$ such that $y_{n} \in U(x)$, $t_{n} \in U(x), t_{n} \in A_{n}$. Then $y_{n} \in U^{2}\left(t_{n}\right) \subset V\left(t_{n}\right) \subset V\left(A_{n}\right)$ which is a contradiction.

Definition 3.2. If $\mathscr{C}$ is a convergence class for $(E, \mathscr{J})$ then $(\mathscr{C}, \mu(\mathscr{C}))$ is said to be monotone complete if and only if each increasing net in $(\mathscr{C}, \mu(\mathscr{C}))$ converges and each decreasing net $\left(A_{n}: n \in D\right)$ for which $\cap A_{n} \neq \phi$ converges.

Theorem 3.4. Let $(E, \mathscr{J})$ be a uniform space. Then

(1) If $f:(E, \mathscr{J}) \rightarrow(E, \mathscr{J})$ is uniformly continuous and $\mathscr{C}$ is any convergence class for $(E, \mathscr{J})$ such that $A \in \mathscr{C}$ implies $f(A) \in \mathscr{C}$ then $g:(\mathscr{C}, \mu(\mathscr{C})) \rightarrow(\mathscr{C}, \mu(\mathscr{C}))$ defined by $g(A)=f(A)$ is uniformly continuous.

(2) If $(\mathscr{C}, \mu(\mathscr{C}))$ is separated and monotone complete, then either,

(a) $g(A)=A$ for some $A \in \mathscr{C}$, or

(b) there exists $A \in \mathscr{C}$ such that $g(A) \subset A$ and $\cap\left(g^{n}(A): n=1,2\right.$, $\cdots)=\phi$ or

(c) $g(A), A$ are not comparable for each $A \in \mathscr{C}$.

Proof. (1) If $f$ is uniformly continuous then $(f \times f) \mathscr{J} \geqq \mathscr{J}$. Then, if $\mathscr{T}(V)$ is a generator of $\mu(\mathscr{C})$, there exists $U \in J$ such that 
$V \supset(f \times f)(U)$. It is an easy computation to show that $(g \times g) \mathscr{T}(U) \subset$ $\mathscr{T}(V)$ so $(g \times g) \mu(\mathscr{C}) \geqq \mu(\mathscr{C})$ and $g$ is uniformly continuous.

(2) If $A \subset g(A)$ for some $A \in \mathscr{C}$, then $A \subset g(A) \subset g^{2}(A) \cdots$ is a monotone net in $\mathscr{C}$ and hence converges to $B \in \mathscr{C}$. By (1) $g(A) \subset g^{2}$ $(A) \subset \cdots$ converges to $g(B)$. Since $(\mathscr{C}, \mu(\mathscr{C}))$ is separated, $B=g(B)$.

If $A \supset g(A)$ for some $A \in \mathscr{C}, A \supset g(A) \supset g^{2}(A) \cdots$ is a decreasing. net in $(\mathscr{C}, \mu(\mathscr{C}))$. If it is true that $\left(g^{n}(A): n=1,2, \cdots\right) \neq \phi$, then $g^{n}(A) \rightarrow B, g^{n}(A) \rightarrow g(B)$ and $B=g(B)$. Hence, if neither (a) nor (b) holds, it must be that $A \not \subset g(A)$ and $g(A) \not \subset A$ for all $A \in \mathscr{C}$. That is, (c) holds.

Recall that if $f:(E, \mathscr{J}) \rightarrow S$ is a bijection, then there is a finest uniform structure for $S$ which makes $f$ uniformly continuous, namely $(f \times f)(\mathscr{J})$.

Definition 3.3. If $\mathscr{C}_{1}, \mathscr{C}_{2}$ are convergence classes for $\left(E_{1}, \mathscr{J}_{1}\right)$, $\left(E_{2}, \mathscr{F}_{2}\right)$ respectively, the natural uniformity $\mu\left[\mathscr{C}_{1}, \mathscr{C}_{2}\right]$ on $\left[\mathscr{C}_{1}, \mathscr{C}_{2}\right]=$ $\left\{A \times B: A \in \mathscr{C}_{1}, B \in \mathscr{C}_{2}\right\}$ is the finest uniform structure on $\left[\mathscr{G}_{1}, \mathscr{C}_{2}\right.$ ] which makes the bijection $f:\left(\mathscr{C}_{1} \times \mathscr{C}_{2}, \mu\left(\mathscr{C}_{1}\right) \times \mu\left(\mathscr{C}_{2}\right)\right) \rightarrow\left[\mathscr{C}_{1}, \mathscr{C}_{2}\right]$ defined by $f(A, B)=A \times B$ uniformly continuous.

THeOREM 3.5. (1) Let $\mathscr{C}_{1}, \mathscr{C}_{2}$ be convergence classes for $(E, \mathscr{J})$. Then $\left(A_{n} \times B_{n}: n \in D\right)$ converges to $A \times B$ in $\left(\left[\mathscr{C}_{1}, \mathscr{C}_{2}\right], \mu\left[\mathscr{C}_{1}, \mathscr{C}_{2}\right]\right)$ if and only if $\left(A_{n}: n \in D\right),\left(B_{n}: n \in D\right)$ converge to $A, B$ in $\left(\mathscr{C}_{1}, \mu\left(\mathscr{C}_{1}\right)\right)$, $\left(\mathscr{C}_{2}, \mu\left(\mathscr{C}_{2}\right)\right)$ respectively.

(2) If $\left(A_{n}: n \in D\right),\left(B_{n}: n \in D\right)$ are nets in $(\mathscr{C}, \mu(\mathscr{C}))$ which converge to $A, B$ respectively and $A_{n} \subset B_{n}$ for $n$ sufficiently large, then $A \subset \operatorname{clos} B$.

Proof. (1) If $\left(A_{n}: n \in D\right) \rightarrow A$ and $\left(B_{n}: n \in D\right) \rightarrow B$, then $\left(A_{n} \times B_{n}\right.$ : $n \in D) \rightarrow A \times B$ by the continuity of the map $f:(A, B) \rightarrow A \times B$. If $\left(A_{n} \times B_{n}: n \in D\right) \rightarrow A \times B$ and $V \in \mathcal{F}$, then, when $\mathscr{T}(V)=\{(S, M): S \subset$ $V(M), M \subset V(S)\}, \mathscr{C}(V)=\{((R, Y),(F, X)):(R, F) \in \mathscr{T}(V),(Y, X) \in$ $\mathscr{T}(V)\}$ is in $\mu\left(\mathscr{C}_{1}\right) \times \mu\left(\mathscr{C}_{2}\right)$. Thus, by definition, $(f \times f) \mathscr{L}(V)(A \times B)=$ $\{R \times Y:(R, A) \in \mathscr{T}(V),(Y, B) \in \mathscr{T}(V)\}$ is a neighborhood of $A \times B$. It follows that $\left(A_{n}, A\right) \in \mathscr{T}(V)$ and $\left(B_{n}, B\right) \in \mathscr{T}(V)$ for $n$ sufficiently large so $\left(A_{n}: n \in D\right) \rightarrow A$ and $\left(B_{n}: n \in D\right) \rightarrow B$.

(2) We have for $V \in \mathscr{J}$, an index $n \in D$ such that $A_{n} \subset B_{n}, A \subset$ $V\left(A_{n}\right), B_{n} \subset V(B)$ so $A \subset V^{2}(B)$ and the result follows from this fact.

The result above, as well as the theorem below will be used in the next section.

THEOREM 3.6. Let $\mathscr{C}$ be a convergence class for the the uniform space $(E, \mathcal{F})$. Then

(1) If $\left(A_{n}: n \in D\right) \rightarrow A$ and $x \in A$, then there exists a directed set 
$H$ and functions $p: H \rightarrow D, m: H \rightarrow E$, such that $p(H)$ is cofinal in $D$, the net $m$ converges to $x$ and $m(h) \in A_{p(h)}$ for all $h \in H$.

(2) If $\left(A_{n}: n \in D\right) \rightarrow A$ and a net $m: H \rightarrow E$ converges to $x$ with $m(h) \in A_{p(h)}, p(H)$ cofinal in $D, p: H \rightarrow D$, then $x \in$ clos $A$.

Proof. (1) Order $D \times \mathscr{J}$ by $(n, V) \geqq(m, U)$ if $n \geqq m$ and $V \subset$ $U$. By convergence, if $(n, V) \in D \times \mathscr{J}$ there exists $p(n, V) \in D$ and $m(n, V) \in A_{p(n, V)}$ such that $p(n, V) \geqq(x, m(n, V)) \in V$. The result follows from this.

(2) If $V \in \mathcal{J}, m(h) \in V(x) \cap A_{p(h)}$ for $h$ sufficiently large. But, by convergence, there is an index $h$ such that $A_{p(h)} \subset V(A)$ also. It follows that for some $a \in A$, some $h \in H,(m(h), x) \in V,(m(h), a) \in V$. Thus $a \in V^{2}(x)$ and the result follows.

4. Fixed point theorem for $\mathscr{C}$-multifunctions, Let $\mathscr{C}$ be a convergence class for the uniform space $(E, \mathscr{J})$. If $F:(E, \mathscr{J}) \rightarrow(\mathscr{C}$, $\mu(\mathscr{C})$ ) is a function, then $F^{n}, n=2,3, \cdots$ is defined inductively as follows. (Notice that $F^{n}(x)$ need not be in $\mathscr{C}$ if $n>1$.) If $x \in E$, $\left.F^{2}(x)=\cup F(y): y \in F(x)\right)$ and $\left.F^{n+1}(x)=\cup F(y): y \in F^{n}(x)\right)$ for $n>2$. If $F^{n}(x) \in \mathscr{C}$ for each $n$ and each $x \in E$, then $F$ is called a $\mathscr{C}$-multifunction.

Definition 4.1. A $\mathscr{C}$-multifunction $F:(E, \mathscr{J}) \rightarrow(\mathscr{C}, \mu(\mathscr{C}))$ is condensing if $F$ is continuous and $V \in \mathscr{J}, x \neq y, x, y \in E$ implies there exists $n=n(x, y, V)$ such that $F^{n}(x) \times F^{n}(y) \subset V$.

EXAMPLE 6. With respect to the hypotheses of the next theorem, we remark that $(\mathscr{C}, \mu(\mathscr{C}))$ can be compact without $\mathscr{C}$ consisting only of closed sets. Let $E$ be the closed unit interval and let $\mathscr{C}$ consist of all subintervals (open, closed, or half open, half closed) of $E$ along with all singleton subsets of $E$. Then $(\mathscr{C}, \mu(\mathscr{C}))$ is compact.

THEOREM 4.1. If $(E, \mathscr{J})$ is compact and Hausdorff, $(\mathscr{C}, \mu(\mathscr{C}))$ is compact and $F:(E, \mathscr{J}) \rightarrow(\mathscr{C}, \mu(\mathscr{C}))$ is condensing, then there exists $x_{0} \in E$ such that $x_{0} \in \operatorname{clos} F\left(x_{0}\right)$.

Proof. Suppose $x \notin F(x)$. Then for some $y \in F(x), y \neq x$. If $V \in$ $\mathscr{J}$, there exists $n(V)$ such that

(1) $F^{n(V)}(x) \times F^{n(V)}(y) \subset V$.

Since $(\mathscr{C}, \mu(\mathscr{C}))$ is compact so is $[\mathscr{C}, \mathscr{C}, \mathscr{C}]$ by Definition 3.3. Hence, with $\mathscr{J}$ directed by reverse inclusion, the net $p$ defined by $p=\left(F^{n(V)}(x) \times\right.$ $\left.F^{n(V)}(y) \times F^{n(V)+1}(x): V \in \mathscr{J}\right)$ has a convergent subnet $t: D \rightarrow[\mathscr{C}, \mathscr{C}, \mathscr{C}]$. If $t \rightarrow A \times B \times T$, then by (1) and Theorem 3.5, $A \times B \subset V$ for each $V \in \mathscr{J}$. Since $(E, \mathscr{J})$ is Hausdorff, $A \times B$ is contained in the 
diagonal of $E \times E$ so $A=B=\left\{x_{0}\right\}$ for some $x_{0} \in E$.

Now $y \in F(x)$ so $F^{n(V)}(y) \subset F^{n(V)+1}(x)$. It follows from Theorem 3.5 that $x_{0} \in \operatorname{clos} T$.

Consider $z \in T$. By Theorem 3.6 and the fact that $t$ is a subnet of $p$, there is a net $m: H \rightarrow E$ and a function $f: H \rightarrow \mathcal{J}$, such that $f(H)$ is cofinal in $\mathscr{J}, m \rightarrow z, m(h) \in F\left(F^{n(f(h))}(x)\right), h \in H$. So,

(2) $m(h) \in F(u(h)), u(h) \in F^{n(f) h)}(x)$.

By compactness of $(E, \mathscr{J}),(u(h): h \in H)$ has a convergent subnet $w$. By (2) $w \rightarrow x_{0}$ and since $F$ is continuous, $F(w) \rightarrow F\left(x_{0}\right)$. By (2), the fact that $m \rightarrow z$, and Theorem 3.5, $z \in \operatorname{clos} F\left(x_{0}\right)$.

In summary we have $T \subset \operatorname{clos} F\left(x_{0}\right)$ and $x_{0} \in \operatorname{clos} T$ so $x_{0} \in \operatorname{clos} F\left(x_{0}\right)$.

COROLLARY 4.1. Let $\mathscr{C}$ be thet set of all non-empty closed subsets of a compact, Hausdorff uniform space $(E, \mathcal{J})$ and let a continuous function $F:(E, \mathscr{J}) \rightarrow(\mathscr{C}, \mu(\mathscr{C}))$ satisfy the following condition: $V \in$ $\mathscr{J}, x \neq y$ implies $F^{n}(x) \times F^{n}(y) \subset V$ for some $n=n(x, y, V)$. Then there is a unique $x_{0} \in E$ such that $x_{0} \in F\left(x_{0}\right)$.

Proof. By results of [7], $F^{n}$ maps $E$ into $\mathscr{C}$ for each $n=1,2$, $3, \cdots$ and $(\mathscr{C}, \mu(\mathscr{C}))$ is compact. Hence, by the previous theorem $x_{0} \in \operatorname{clos} F\left(x_{0}\right)=F\left(x_{0}\right)$ for some $x_{0} \in E$. If also $x \in F(x)$, then given $V \in \mathcal{J}$, it is true that $\left(x, x_{0}\right) \in F^{n}(x) \times F^{n}\left(x_{0}\right) \subset V$ for some $n$. It follows that $\left(x, x_{0}\right) \in \cap\{V: V \in \mathscr{J}\}, x \neq x_{0}$, which contradicts the fact that $(E, \mathcal{J})$ is Hausdorff.

Corollary 4.2. (Bailey [1]). Let $(E, d)$ be a compact metric space and $f:(E, d) \rightarrow(E, d)$ a continuous function such that if $x \neq y$, there exists $n=n(x, y)$ such that $d\left(f^{n}(x), f^{n}(y)\right)<d(x, y)$. Then $f$ has a unique fixed point.

Proof. Under the hypothesis of the theorem it is easy to see that if $\delta>0$ is given and $x \neq y$, there exists $n=n(x, y, \delta)$ such that $d\left(f^{n}(x), f^{n}(y)\right)<\delta$. Then, with $\mathscr{J}$ the natural uniform structure induced by $d$, the hypotheses of Corollary 4.1 are satisfied for $f$ and $(E, \mathscr{J})$ so the result follows.

Now let $(E, d)$ be a bounded metric space and $\mathscr{C}$ any class of nonempty subsets of $E$. The well-known Hausdorff function $h$ on $\mathscr{C}$ is defined by $h(a, b)=\max \{m(A, B), m(B, A)\}$ where $m(A, B)=\sup \{d(x$, $B): x \in A\}$ and $d(x, B)=\inf \{d(x, y): y \in B\}$.

THEOREM 4.2. Let $(E, d)$ be a bounded metric space and let $\mathscr{C}$ be any nonempty class of nonempty subsets of $E$. Let $\mathscr{C}$ satisfy $\left(B_{3}\right)$ of $\S 3$ with respect to the natural uniform structure on $E$ generated by the $V_{\delta}^{\prime} ' s, V_{\delta}=\{(x, y): d(x, y)<\delta\}$. Then $(\mathscr{C}, \mu(\mathscr{C}))$ is uniformly metrizable 
and one metric for $(\mathscr{C}, \mu(\mathscr{C}))$ is the Hausdorff function on $\mathscr{C}$.

Proof. If $h(A, B)=0$, then $m(A, B)=m(B, A)=0$. Given $\delta>0$, it follows that $A \subset V_{\hat{o}}(B)$ and $B \subset V_{\hat{o}}(A)$. Since $\left(B_{3}\right)$ holds, $A=B$. Clearly $h(A, B)=h(B, A)$ and, if $A=B, h(A, B)=0$.

To prove the triangle inequality it suffices to show that $m(A, B) \leqq$ $m(A, X)+m(X, B)$ for each $A, B, X \in \mathscr{C}$. Let $\delta>0$ be given.

(1) $m(A, B)<d\left(a_{0}, a\right)+d\left(a, x_{0}\right)+d(x, b)+\delta$ for some $a_{0} \in A$ and all $a \in A, x \in X, b \in B$.

Also $m(A, X) \geqq d(a, X)$ for all $a \in A$ so given $a_{0} \in A$,

(2) there exists $x_{1} \in X$ such that $m(A, X)>d\left(a_{0}, x_{1}\right)-\delta$; similarly

(3) $m(X, B)>d\left(x_{1}, b_{1}\right)-\delta$ for some $b_{1} \in B$. Combining (3), (2), and (1) we have $m(A, X)+m(X, B)>m(A, B)-3 \delta$ and it follows that $m(A, B) \leqq m(A, X)+m(X, B)$.

We have shown that $h$ is a metric on $\mathscr{C}$. Now let $U_{o} \in \mathscr{J}(h)$, $\mathscr{J}(h)$ the structure on $\mathscr{C}$ generated by $h$. A computation shows that if $(A, B) \in U_{\hat{o}}$ then $A \subset V_{2 \hat{\delta}}(B)$ and $B \subset V_{2 \hat{o}}(A), V_{2 \hat{o}}=\{(x, y): d(x, y)<2 \delta\}$, hence $U_{\hat{o}} \subset \mathscr{T}\left(V_{2 \hat{o}}\right)$ so $\mathscr{J}(h) \geqq \mu(C)$. Similarly $\mathscr{I}\left(V_{\hat{o}}\right) \subset U_{2 \hat{o}}$ so then $\mathscr{J}(h) \leqq \mu(C)$.

The author wishes to thank the referee for several helpful suggestions.

\section{REFERENCES}

1. D. F. Bailey, Somes theorems on contractive mappings, J. London Math. Soc., 41 (1966), 101-106.

2. G. Choquet, Convergences, Ann. de l'University de Grenoble 23 (1947-48), 55-112.

3. Allan C. Cochran, On Uniform Convergence Structures and Convergence Spaces, Doctoral Dissertion, Univ. Oklahoma, 1966.

4. C. H. Cook and H. R. Fischer, Uniform convergence structures, Math. Ann., 173 (1967), 290-306.

5. H. R. Fischer, Limeräume, Math. Ann., 137 (1959), 269-303.

6. H. H. Keller, Die Lime Uniformiserbarkeit des Limesraumes, Math. Ann., 176 (1968), 334-341.

7. E. Michael, Topologies on spaces of subsets, Trans. Amer. Math. Soc., 71 (1955), 151-180.

8. C. D. Richardson, Completions of uniform convergence spaces, Proc. Amer. Math. Soc., 29 (1971), 159-164.

Received September 10, 1970 and in revised form Nay 30, 1972. 


\section{PACIFIC JOURNAL OF MATHEMATICS}

\section{EDITORS}

\section{H. SAMElson}

Stanford University

Stanford, California 94305

C. R. Новву

University of Washington

Seattle, Washington 98105
J. DugunduI

Department of Mathematics University of Southern California

Los Angeles, California 90007

RICHARD ARENS

University of California

Los Angeles, California 90024

\section{ASSOCIATE EDITORS}
E. F. BECKENBACH
B. H. NeUmanN
F. WOLF
K. YosHIDA

\section{SUPPORTING INSTITUTIONS}

UNIVERSITY OF BRITISH COLUMBIA

UNIVERSITY OF SOUTHERN CALIFORNIA

CALIFORNIA INSTITUTE OF TECHNOLOGY

STANFORD UNIVERSITY

UNIVERSITY OF CALIFORNIA

UNIVERSITY OF TOKYO

MONTANA STATE UNIVERSITY

UNIVERSITY OF UTAH

UNIVERSITY OF NEVADA

WASHINGTON STATE UNIVERSITY

NEW MEXICO STATE UNIVERSITY

OREGON STATE UNIVERSITY

UNIVERSITY OF OREGON

OSAKA UNIVERSITY

UNIVERSITY OF WASHINGTON

* *

AMERICAN MATHEMATICAL SOCIETY

NAVAL WEAPONS CENTER 


\section{Pacific Journal of Mathematics}

\section{Vol. 43, No. $1 \quad$ March, 1972}

Alexander (Smbat) Abian, The use of mitotic ordinals in cardinal

arithmetic ....................................... 1

Helen Elizabeth. Adams, Filtrations and valuations on rings ......... 7

Benno Artmann, Geometric aspects of primary lattices .............. 15

Marilyn Breen, Determining a polytope by Radon partitions ........... 27

David S. Browder, Derived algebras in $L_{1}$ of a compact group .......... 39

Aiden A. Bruen, Unimbeddable nets of small deficiency .............. 51

Michael Howard Clapp and Raymond Frank Dickman, Unicoherent

compactifications ............................... 55

Heron S. Collins and Robert A. Fontenot, Approximate identities and the strict topology ................................... 63

R. J. Gazik, Convergence in spaces of subsets................. 81

Joan Geramita, Automorphisms on cylindrical semigroups ........... 93

Kenneth R. Goodearl, Distributing tensor product over direct product ..... 107

Julien O. Hennefeld, The non-conjugacy of certain algebras of

operators ................................... 111

C. Ward Henson, The nonstandard hulls of a uniform space ........... 115

M. Jeanette Huebener, Complementation in the lattice of regular

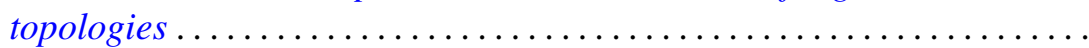

Dennis Lee Johnson, The diophantine problem $Y^{2}-X^{3}=A$ in a

polynomial ring .................................... 151

Albert Joseph Karam, Strong Lie ideals . . . . . . . . . . . . . . . . . . . . 157

Soon-Kyu Kim, On low dimensional minimal sets ............... 171

Thomas Latimer Kriete, III and Marvin Rosenblum, A Phragmén-Lindelöf

theorem with applications to $M(u, v)$ functions ..... . .

William A. Lampe, Notes on related structures of a universal algebra . . . . 189

Theodore Windle Palmer, The reducing ideal is a radical .

207

Kulumani M. Rangaswamy and N. Vanaja, Quasi projectives in abelian and module categories ................................ 221

Ghulam M. Shah, On the univalence of some analytic functions ......... 239

Joseph Earl Valentine and Stanley G. Wayment, Criteria for Banach

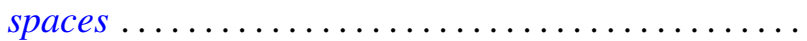

Jerry Eugene Vaughan, Linearly stratifiable spaces ............... 253

Zbigniew Zielezny, On spaces of distributions strongly regular with respect to partial differential operators ..................... 